\title{
Explicit Constructive Logic ECL: a New Representation of Construction and Selection of Logical Information by an Epistemic Agent
}

\author{
Paolo Gentilini ${ }^{1}$ and Maurizio Martelli ${ }^{2}$ \\ 1 DIMA, University of Genoa, Via Dodecaneso 35, 16146 Genova, and \\ IMATI-CNR, via de Marini 6, 16149 Genova, Italy \\ 2 DIBRIS, University of Genoa, Via Dodecaneso 35, 16146 Genova, Italy
}

\begin{abstract}
One of the seminal goals of Explicit Constructive Logic (ECL) is to provide a constructive formulation of full higher order logic (Classical Type Theory $\mathbf{L} \mathbf{K}_{\omega}$ ) that can be seen as a foundation for knowledge representation. Moreover, the development of this work has produced the basis of a new approach to constructivism in Logic.ECL is introduced as a sub-system $\mathbf{Z}_{\omega}$ of $\mathbf{L} \mathbf{K}_{\omega}$. Also the first order case $\mathbf{Z} \mathbf{1}$ and the propositional case $\mathbf{Z P}$ of $E C L$ are examined. A comparison between $E C L$ 's constructivism and the corresponding features of Intuitionistic Logic, and Constructive Paraconsistent Logic is proposed.
\end{abstract}

Keywords: Constructivism in Logic, Higher Order Logic, Intuitionistic and Constructive Paraconsistent Logic

\section{Introduction}

Full higher order logic can be an extremely powerful tool for knowledge representation if some of its features could be simplified and controlled. A constructive formulation of it is the goal of Explicit Constructive Logic (ECL) and will be presented in this paper. We will start from the sequent version $\mathbf{L} \mathbf{K}_{\boldsymbol{\omega}}$ of Classical Type Theory as presented in [7] where the Church formalism is used and logical connectives are expressed as typed formulas.For the extensive definitions of the syntax of typed language and the basic notions of proof-theory (sequents, rules, proof-trees and so on) see [7] Sections 2 and 3. To realize the foundational principles of the $E C L$ inference we will define the systems $\mathbf{Z}_{\omega}$ and $\mathbf{Z}_{\omega}^{*}$, (included in $\mathbf{L K}_{\boldsymbol{\omega}}$ ), that, even maintaining a very high expressive power, show strong constructivity properties and could admit a new organization of proofs (through a Normal Form Theorem). $\mathbf{Z}_{\omega}$ could be also seen as a generalization, at the theoretical level, of the features of Higher Order Uniform Logic, introduced in [8] to express Higher Order Logic Programming. Indeed, in both cases the very specific behaviour of proofs is that the principal formula in the conclusion of a logical rule can be deduced only if some constraints on the introductions of the auxiliary formulas in the rule premise(s) are respected. We will also examine the first order case $\mathbf{Z} \mathbf{1}$ and the propositional case $\mathbf{Z P}$ of $E C L$. The real novelty 
of any proposed new logical framework must arise clearly at the propositional level, and this is the case for Intuitionistic Logic and Paraconsistent Logic. The Church formalism is maintained also for the first order case and the propositional case, since it is very convenient for a deep analysis of logical connectives. A parallel and a comparison between $E C L$ and Intuitionistic Logic and Paraconsistent Logic as different kinds of constructive logics will be often proposed in this paper. The constructivity of Intuitionistic Logic [10] doesn't need explanations. As to the constructivity of Paraconsistent Logic we consider only a well delimited area of paraconsistency, given by the Logics of Formal Inconsistency (LFI) and the included $C$-system family, introduced in [2]. The formal notion of constructive paraconsistent logic is introduced in [6].

\section{Explicit Logical Constructivism: Syntactic Environment and Epistemological Basis}

We will now give a short synthesis of the epistemological basis of Explicit Constructive Logic: the style will be heuristic and intuitive.

We will use the logical connectives à la Church [3]:

$$
\left\{\neg_{o \rightarrow o}, \wedge_{o \rightarrow(o \rightarrow o)}, \vee_{o \rightarrow(o \rightarrow o)}, \supset_{o \rightarrow(o \rightarrow o)}, \forall_{(\alpha \rightarrow o) \rightarrow o}, \exists_{(\alpha \rightarrow o) \rightarrow o}, \perp_{o}, \top_{o}\right\}
$$

where the type subscript is in general omitted in writing formulas. We call judgments of the epistemic subject the compound logical formulas: we want to convey the idea that logical information is provided by compound propositions and is introduced by the subject starting from elementary data, expressed by atomic formulas. The elementary data reflect the elementary facts that take place inside a fixed empirical world that is assumed as reference for constructing knowledge. The difference in the logical and epistemic role between atomic formulas and compound formulas is so relevant, that we introduce for it specific meta-symbols: thus, latin capital letters $A, B, C, \ldots$ will indicate arbitrary formulas, whereas latin capital letters with the + superscript $A^{+}, B^{+}, C^{+}, \ldots$ will indicate arbitrary atomic formulas. We recall that logical connectives are not atomic formulas. A formula is atomic if the outermost symbol is not a logical connective. A formula is an atom if it has not proper Church sub-term. In particular, the $o$-typed logical connectives, i.e. the logical constants $\perp_{o}, \top_{o}$ are atoms but not atomic formulas. The first expresses the judgement which is always acceptable (for which no criticism is possible) so that the sequent $\vdash T$ is always provable; the latter expresses the judgement which is always refutable (for which no corroboration is possible) so that the sequent $\perp \vdash$ is always provable. (see also [7], Sec. 2 and 3). A further technical remark is that in this paper we will always use the sequent version of the considered logical systems. Other foundational assumptions are the following. In the demonstration (argumentation) produced by the epistemic subject, judgments are not received from the external world: they must be explicitly constructed alongside the demonstration itself. Only elementary data are necessarily provided by the external world. Therefore, logical 
information has to be always reconstructed by the epistemic subject and never merely acquired; differently, elementary data are merely acquired. Simmetrically, judgements, alongside a demonstration, cannot be eliminated without an explicit logical motivation: this must have the form of another explicit judgment of the espistemic subject, that is by applying a logical rule. The eliminating rules, in a context where the cut rule in strictly bounded and anyway eliminable (as it happens in the main logical calculi) are the so called Comprehension rules (Comp rules) in [7], i.e $\forall-L$ and $\exists-R$. An exhaustive discussion of the elimination power of Comp Rules is given in [7] Section 3. Thus, the full higher order system $\mathbf{Z}_{\omega}$ for $E C L$ Logic that we are going to define, is a subsystem of $\mathbf{L} \mathbf{K}_{\boldsymbol{\omega}}$ where:

- only atomic formulas occur in the logical axioms;

- weakening rules are admitted only to introduce atomic formulas, i.e. weakening cannot introduce logical information;

- cut rule is admitted only with atomic cut formulas, i.e. cut cannot eliminate logical information;

- each logical rule is always thought as occurring in some proof $\mathrm{P}$, and have constraints on its auxiliary formulas that take into account their introduction in the whole proof-segment above the premise(s) of the rule occurrence in P. That is, they are global and not local constraints. The underlying idea is that the construction of a judgment introducing new logical information must be based only on previously produced logical information which has been itself acceptably constructed.

We note that the restrictions on weakening and cut rules immediately follow from the epistemic assumptions mentioned above. The constraints on the logical rules will be presented and discussed in detail in the next Sections. Moreover, the constructivity properties we assign to $\mathbf{Z}_{\omega}$ are also justified through the comparison with those logics that in the literature are already considered as constructive (Intuitionistic Logic, Paraconsistent Logic, Uniform Logic).

The constructivism of Intuitionistic Logic $\mathbf{L} \mathbf{J}$ is well known. We recall that, from a merely technical point of view, it is characterized by the refutation of the excluded middle (or tertium non datur) principle, syntactically expressed by the schema $A \vee \neg A$ and, collaterally, also by the refutation of the left double negation principle, expressed by the schema $\neg \neg A \supset A$. The standard sequent version $\mathbf{L} \mathbf{J}$ is characterized by the following condition: each sequent in a proof has the empty set or a singleton as succedent. However, this is not strictly necessary: in the sequent version of Maehara [9] p. 52, such condition is replaced by local contraints on three logical rules, among which, centrally, the negation rule on the right $\neg-R$. Even if intuitionism has many peculiar constructive features, that cannot be reduced to the mere refutation of tertium non datur, it is a fact that LJ plus $\vdash A \vee \neg A \equiv$ classical LK.

The constructivism of Paraconsistent Logic has been only recently defined in a formal way, and the set of paraconsistent logics to which the notion can be applied must be clearly delimited. We consider here the system CI examined in [6]. Paraconsistent Logic arises form the refutation of the classical (syntactic) principle ex contraditione quodlibet that can be expressed by the LK-provable 
sequent schema $A \wedge \neg A \vdash$ which is classically equivalent to the non contradiction principle $\vdash \neg(A \wedge \neg A)$. CI and the various systems in the $C$-system family do not prove contradictions, but can support axioms of the form $B \wedge \neg B$ without trivializing, i.e. without proving the empty sequent " $\vdash$ ". Even if the refutation of ex contradictione quodlibet could seem today a position which is naturally constructive, in [6] a formal definition of constructive paraconsistent logic is given, also employing the introduction of antisimmetry connections between $C$ system paraconsistency and intuitionistic logic.

The Uniform Logic introduced in [8], that we indicate here with $\mathbf{L} \mathbf{U}_{\boldsymbol{\omega}}$, is a sub-system of Higher Order Intuitionistic Logic $\mathbf{L} \mathbf{J}_{\boldsymbol{\omega}}$ where a particular constraint is added for the application of logical rules inside a proof-tree. Essentially, if a logical rule occurrence $\mathcal{R}$ has the auxiliary formula(s) in the premise succedent(s), then it (they) must be the principal formula(s) of the logical rule occurrence $(s)$ immediately above $\mathcal{R}$ in the branch. Uniform Logic specifies the intuitionism constructivity in the direction of computation: indeed, it expresses abstract logic programming languages. Moreover, as to the main discussion of this paper, it must be remarked that the rule-constraint mentioned above is a first example of global, i.e. referred to the context of the rule-occurrence in the proof, and not local constraint.

As detailed later, $\mathbf{Z}_{\omega}$ shares relevant specific features with Intuitionistic Logic, since it does not prove both $\aleph_{0}$ instances of excluded middle principle $A \vee \neg A$ and $\aleph_{0}$ instances of left double negation principle $\neg \neg A \supset A$. It could be called also a pseudo-intuitionistic system (such notion is formally introduced in $[6])$.

$\mathbf{Z}_{\omega}$ shares relevant specific features with Paraconsistent Logic, since it does not prove $\aleph_{0}$ instances of non contradiction principle $\neg(A \wedge \neg A)$ and can be extended by $\aleph_{0}$ contradictions without trivializing. It could be called also a paraconsistent system.

$\mathbf{Z}_{\omega}$ shares some properties with Uniform Logic. In fact it does not hold, (in general, for $\mathbf{Z}_{\omega}$ proof trees), the possibility of any permutation of the order of propositional rules in a proof branch without changing the end-sequent, even in those cases where classical logic would admit such a permutation.

We also point out that $E C L$ has a strong expression power. Indeed, besides the $\mathbf{Z}_{\omega}$-proof capabilities allowed by the higher order, the following properties also hold for $\mathbf{Z} \mathbf{1}$ and $\mathbf{Z P}$ :

$\mathbf{Z}_{\omega}$ proves $\aleph_{0}$ arbitratrily complex instances of excluded middle principle $A \vee$ $\neg A$ that $\mathbf{L J}_{\boldsymbol{\omega}}$ does not prove;

$\mathbf{Z}_{\omega}$ proves $\aleph_{0}$ arbitratrily complex instances of non contradiction principle $\neg(A \wedge \neg A)$ that $\mathbf{C I}_{\omega}$ does not prove.

\section{The Systems $\mathrm{Z}_{\omega}, \mathrm{Z1}, \mathrm{ZP}$ for Explicit Constructive Logic (ECL)}

In the sequel we briefly call bottom and top the formulas $\perp$ and $T$, recalling that they are not atomic formulas. Moreover, if $\mathbf{Z}_{\omega}$ proves the sequent $\vdash B$ we say that 
$B$ is a theorem of $\mathbf{Z}_{\omega}$, if $\mathbf{Z}_{\omega}$ proves the sequent $E \vdash$ we say that $E$ is a refuted by $\mathbf{Z}_{\omega}$. The language of $\mathbf{Z}_{\omega}$ is that of $\mathbf{L} \mathbf{K}_{\boldsymbol{\omega}}$ (see [7] Sec. 2) with the exclusion of all the equality symbol $=_{o \rightarrow(o \rightarrow o)}$ that would express the equality relation between $o$-typed formulas, since such relation is not considered by ECL. For the notions concerning general proof theory, the analysis of proofs as well as the notions of ancestor, descendant, auxiliary formula, principal formula and so on, we refer to [7] Section 2 and 6.

The sequent system $\mathbf{Z}_{\omega}$ is the following:

(In a sequent $\Omega, \Delta, \Gamma, \Pi, \Theta, \ldots$ will be used as meta-expressions for finite and possibly empty sets of $o$-typed formulas, $A, B, C, D$, ...for arbitrary isolated formulas in a sequent, $A^{+}, B^{+}, C^{+}, D^{+}, \ldots$ for arbitrary atomic isolated formulas. The writing $\Omega, \Delta$ denotes $\Omega \cup \Delta$ )

\section{Axioms}

Logical axioms $\quad A^{+} \vdash A^{+}$with the following constraint:

if the atomic $A^{+}$is a $\beta$-redex, possible repeated application of the $\lambda$-rule does not produce any $\beta$-contractum $F$ which is a descendant of $A^{+}$and is a non-atomic formula.

$\begin{array}{lc}\text { Top axiom } & \vdash \top \\ \text { Bottom axiom } & \perp \vdash \\ \text { Rules } & \end{array}$

\section{Strong Logical Rules:}

Propositional rules:

$$
\begin{array}{lc}
\frac{A, B, \Gamma \vdash \Delta}{A \wedge B, \Gamma \vdash \Delta} \wedge-L & \frac{\Gamma \vdash \Delta, A \quad A \vdash X, B}{\Gamma, \Lambda \vdash \Delta, X, A \wedge B} \wedge-R \\
\frac{\Gamma \vdash \Delta, A, B}{\Gamma \vdash \Delta, A \vee B} \vee-R & \frac{A, \Gamma \vdash \Delta \quad B, \Lambda \vdash X}{A \vee B, \Gamma, \Lambda \vdash \Delta, X} \vee-L \\
\frac{A, \Gamma \vdash \Delta, B}{\Gamma \vdash \Delta, A \supset B} \supset-R & \frac{\Gamma \vdash \Delta, A \quad B, \Lambda \vdash X}{A \supset B, \Gamma, \Lambda \vdash \Delta, X} \supset-L \\
\frac{\Gamma \vdash \Delta, A}{\neg A, \Gamma \vdash \Delta} \neg-L & \frac{A, \Gamma \vdash \Delta}{\Gamma \vdash \Delta, \neg A} \neg-R
\end{array}
$$

It can be noted that the forms of $\wedge-L$ and $\vee-R$ are not the standard one. This is a specific requirement of the Explicit Constructive Logic that will be discussed in the next Sections.

$$
\begin{array}{ll}
\text { Quantifier rules: } \\
\frac{\left[t_{\alpha} / x_{\alpha}\right] A, \Gamma \vdash \Delta}{\forall x_{\alpha} A, \Gamma \vdash \Delta} \forall-L & \frac{\Gamma \vdash \Delta,\left[b_{\alpha} / x_{\alpha}\right] A}{\Gamma \vdash \Delta, \forall x_{\alpha} A} \forall-R \\
\frac{\left[b_{\alpha} / x_{\alpha}\right] A, \Gamma \vdash \Delta}{\exists x_{\alpha} A, \Gamma \vdash \Delta} \exists-L & \frac{\Gamma \vdash \Delta,\left[t_{\alpha} / x_{\alpha}\right] A}{\Gamma \vdash \Delta, \exists x_{\alpha} A} \exists-R
\end{array}
$$

where: in $\forall-L, \exists-R, t_{\alpha}$ is an arbitrary term and in the corresponding $\forall x_{\alpha} A$, $\exists x_{\alpha} A, t_{\alpha}$ may still occur, that is $t_{\alpha}$ may be not fully quantified in $\forall x_{\alpha} A, \exists x_{\alpha} A$; on the other hand, in $\forall-R, \exists-L$, the free variable $b_{\alpha}$ occurring in $\left[b_{\alpha} / x_{\alpha}\right] A$ is uniformly replaced in $\forall x_{\alpha} A, \exists x_{\alpha} A$ by the bound variable $x_{\alpha}$ having the same index, and $b_{\alpha}$ does not occur in $\Gamma, \Delta . b_{\alpha}$ is the proper variable or eigenvariable of the rule.

$$
\lambda \text {-rule: } \quad \frac{\Gamma \prime \vdash \Delta \prime}{\Gamma \vdash \Delta} \lambda
$$


where the sets $\Gamma$ and $\Gamma^{\prime}$ and the sets $\Delta$ and $\Delta /$ differ only in that zero or 1 formula in them is replaced by some formula to which it is $\beta$-reducible. Note that the rule is defined so that the $\beta$-reduction may work either upwards or downwards. We observe that differently from the more usual version (see e.g. [8], [4]) it is imposed here that $\lambda$-rule works on 1 auxiliary formula only, and not simultaneously on any arbitrary set of auxiliary formulas. This option is more coherent with the $E C L$ perspective and with the inclusion of the rule among the strong logical rules, where the control of all the origins of the auxiliary formulas of the rule-occurrence in the above standing proof-segment is required. Finally, the inclusion of $\lambda$-rule among strong logical rules is due to the possibility that a rule occurrence $\mathcal{R}$ in a proof $\mathrm{P}$ of $\mathbf{Z}_{\omega}$ may $\beta$-reduce a $\beta$-redex to a non-atomic formula $D$ arbitrarily complex, with a main logical connective (the outermost symbol of $D$ ) that can be seen as introduced by $\mathcal{R}$.

Strong logical rules must fulfil the following constraints:

- If $\mathcal{R}$ is a 1 premise strong logical rule then each occurrence of $\mathcal{R}$ in a $\mathbf{Z}_{\omega}$-proof $\mathrm{P}$ is such that at least 1 auxiliary formula has at least 1 uppermost ancestor introduced by an axiom.

- If $\mathcal{R}$ is a 2 premise strong logical rule, having i.e. two auxiliary formulas, then each occurrence of $\mathcal{R}$ in a $\mathbf{Z}_{\omega}$-proof $\mathrm{P}$ is such that each auxiliary formula has at least 1 uppermost ancestor introduced by an axiom.

- If $\mathcal{R}$ is a $\lambda$-rule then each $\mathcal{R}$-occurrence in a proof $\mathrm{P}$ in $\mathbf{Z}_{\omega}$ is such that its auxiliary formula has at least 1 uppermost ancestor introduced by an axiom.

Weak Logical Rules

\section{bottom rule $\frac{\perp \vdash}{\perp \vdash A}$}

where $A$ is an arbitrary formula without sub-formulas of the form $\forall x_{o}\left(x_{o}\right)$, $\exists x_{o}\left(x_{o}\right)$.

top rule:

$$
\frac{\vdash \top}{B \vdash \top}
$$

where $B$ is san arbitrary formula without sub-formulas of the form $\forall x_{o}\left(x_{o}\right)$, $\exists x_{o}\left(x_{o}\right)$.

Structural Rules

Wakening rules:

$$
\begin{array}{cc}
\frac{\Gamma \vdash \Delta}{\Gamma \vdash \Delta, A^{+}} W 1-R & \frac{\Gamma \vdash \Delta}{A^{+}, \Gamma \vdash \Delta} W 1-L \\
\frac{\vdash}{\vdash} W 2-R \quad \frac{\vdash}{F \vdash} W 2-L \\
\frac{\Gamma \vdash \Delta, B^{+} B^{+}, \Gamma \vdash \Delta}{\Gamma \vdash \Delta} C u t 1 & \frac{\vdash F \quad F \vdash}{\vdash} \text { Cut } 2
\end{array}
$$

Cut Rules

Structural rules must fulfil the following constraints:

- Each W1 -principal formula is atomic, and in any W1-rule at least 1 set of the contex is non-empty.

- Each W2- principal formula may be arbitrary.

- Cut1-formula $B^{+}$is atomic, such that if it is a $\beta$-redex, possible repeated application of the $\lambda$-rule does not produce any $\beta$-contractum $G$ which is a descendant of $B^{+}$and is a non-atomic formula. Moreover, at leaast 1 context set is non-empty.

- Cut2-formula F may be arbitrary. 


\subsection{The First Order System Z1 of ECL}

The language of $\mathrm{Z} 1$ is defined as follows:

The well formed expressions of $\mathbf{Z} \mathbf{1}$ are Church-terms with the following constraints:

- variables are only of type $i$;

- $\lambda$-abstractions are only over variables of type $i$ and on formulas of type $o$, i.e. have only the form $\lambda x_{i} A_{o}$ with type $i \rightarrow o$;

- quantifiers occur only with type $(i \rightarrow o) \rightarrow o$, i.e. with the forms $\exists_{(i \rightarrow o) \rightarrow o}$ $\forall(i \rightarrow o) \rightarrow o$;

- if $\tau$ is a type occurrence in any $\mathbf{Z} \mathbf{1}$-espression, no occurrences of the type $o$ in $\tau$ precede any occurrence of the type $i$ in $\tau$;

- non-logical constants of $\mathbf{Z 1}$ are only of a type $\tau$ such that:

either in $\tau$ the type $o$ does not occur, or the type $o$ has at most one occurrence as tail of $\tau$; $\tau$ has a condensed writing of the form: $i \rightarrow i \rightarrow i \rightarrow \ldots \rightarrow u$, where $u$ is a primitive type.

Deduction apparatus of $\mathrm{Z} 1$ :

It is identical to $\mathbf{Z}_{\omega}$ deduction apparatus, with the constraint that rules are restricted to sequents of $\mathbf{Z} \mathbf{1}$-formulas, so that only $i$-typed terms can be quantified. The $\lambda$-rule could be useful but is not strictly necessary for the expressivity of the system. Thus, we denote $\mathbf{Z} \mathbf{1} \lambda$ the version including $\lambda$-rule, $\mathbf{Z} \mathbf{1}$ the $\lambda$-rule free version.

\subsection{The Propositional Calculus ZP of ECL}

The language of $\mathbf{Z P}$ is obtained from $\mathbf{Z 1}$ with the following restrictions:

-quantifiers, variables, $\lambda$-abstraction expressions, do not occur in the language;

- the only non logical constants are o-typed atoms, also called propositional letters.

The deduction apparatus is obtained from that of $\mathbf{Z 1}$ by deleting quantifier rules.

3.3 Immediate Properties and Definitions of $\mathrm{Z}_{\omega}, \mathrm{Z} 1, \mathrm{ZP}$

In this Section we will focus mainly on the most powerful and expressive system, which is $\mathbf{Z}_{\omega}$; however, many definitions and properties naturally extend to $\mathbf{Z} \mathbf{1}$ and $\mathbf{Z P}$.

Remark 1. Weak logical rules are also imposed by the necessity to give a suitable proof power to $E C L$ systems. For example, in $\mathbf{L} \mathbf{K}_{\boldsymbol{\omega}}$, the axiom $\perp \vdash$ makes superfluous a rule of the form

$\frac{\perp \vdash}{\perp, U \vdash V}$

$U, V$ arbitrary sets, due to constraint free weakening rules of $\mathbf{L} \mathbf{K}_{\boldsymbol{\omega}}$. In $E C L$ systems, such approach would not be conceivable.

Definition 1. Let $\mathrm{P}$ be a proof tree in $\mathbf{Z}_{\omega}$. Then we say that the occurrence of the formula $\mathbf{A}$ in $P$ is strongly introduced if it is integral descendant of an axiom formula or it is integral descendant of the principal formula of a strong logical rule. We say that the occurrence of the formula $\mathbf{A}$ in $P$ is weakly introduced if 
it is integral descendant of the principal formula of a weak logical rule or of a weakening formula.

For the definition of integral descendant of a formula occurrence in a proof see [7] Def. 6.5.ii p. 750. Intuitively the integral descendant $B$ of the formula occurence $F$ in a proof branch is such that $B$ and $F$ are occurrences of the same formula, i.e. $B \equiv F$, connected by a proof-path where $F$ (or $B$ ) is never an auxiliary formula of any rule.

Definition 2. Among the strong logical rules we call major (strong) logical rules those where all auxiliary formulas must be strongly introduced, minor (strong) logical rules those where at least 1 auxiliary formula may be weakly introduced.

Corollary 1. $\{\bigvee-L, \wedge-R, \supset-L, \neg-R, \neg-L, \forall-L, \forall-R, \exists-L, \exists-R$, $\lambda$-rule $\}$ is the set of major logical rules in $\mathbf{Z}_{\omega},\{\vee-R, \wedge-L, \supset-R\}$ is the set of minor logical rules in $\mathbf{Z}_{\omega}$.

Definition 3. Let $\mathrm{P}$ be a proof tree in $\mathbf{Z}_{\omega}$. Then we say that a sequent $S$ occurring in $P$ is strongly proven in $P$ if each formula of $\mathrm{S}$ is strongly introduced in $\mathrm{P}$. We say that $S$ is weakly proven in $P$ otherwise.

Caveat: the same $S$ may be strongly proven in a proof $\mathrm{P}$ and, simultaneously, weakly proven in a different proof Q. In ECL logic the proof-context of a sequent or of a formula has a substantial role, and this is coherent with the fact that the constraints on the application of a logical rule in ECL are always global and not local. We also mention these two evident facts: $\mathbf{Z}_{\omega}$ is consistent, since it is a $\mathbf{L} \mathbf{K}_{\boldsymbol{\omega}}$ subsystem; moreover, if $\mathrm{P}$ is a $\mathbf{Z}_{\omega}$-proof, it cannot have an end-sequent where all formulas are weakly introduced.

\section{General Epistemological and Logical Justifications for Axioms and Rules of $\mathrm{Z}_{\omega}, \mathrm{Z} 1, \mathrm{ZP}$, and Further Properties of Connectives and Rules in ECL}

\subsection{The Formulas Bottom and Top}

$o$-typed logical constants $\perp$ and $T$ standardly occur in the Church presentation of Type Theories ([7], [8], [4]). In the Explicit Constructive Logic ECL T expresses the judgment that the subject thinks as always acceptable, beyond any possible confutation, and $\perp$ expresses the judgment that the subject thinks as always refutable, beyond any possible corroboration. That's why, in general, we can state: if $B$ is a $\mathbf{Z}_{\omega}$-theorem different from $\top$, then it is not provable in $\mathbf{Z}_{\omega}$ the sentence $(\top \supset B) \wedge(B \supset \top)$ (or $B \longleftrightarrow \top$ ), and if $E$ is a $\mathbf{Z}_{\omega}$-refuted different from $\perp$, then it is not provable in $\mathbf{Z}_{\omega}$ the sentence $(\perp \supset E) \wedge(E \supset \perp)$ (or $E \longleftrightarrow \perp$ ). In particular, as to the conjunctions that would give the mentioned $\mathbf{Z}_{\omega}$-logical equivalences, it is not provable, in general, in the first case the conjunct $\top \supset B$ and in the second case the conjunct $E \supset \perp$. Remarkable exceptions may exist. For example $T \wedge T$ is a theorem and $T \supset \top \wedge T$ is provable. However, 
for each atomic $A^{+}, A^{+} \vee \neg A^{+}$is a theorem but $\top \supset A^{+} \vee \neg A^{+}$is not provable. For example, for each atomic $B^{+}, \perp \wedge B^{+}$is a refuted and $\perp \wedge B^{+} \supset \perp$ is provable. However, for each atomic $B^{+}, B^{+} \wedge \neg B^{+}$is a refuted but $B^{+} \wedge \neg B^{+} \supset \perp$ is not provable. These examples suggest that explicit constructivity includes $a$ criticism to classical implication. Moreover, the usual systems of classical, intuitionistic and paraconsistent logics lack such fine separation capability: all of them make top particles equivalent to theorems, and bottom particles equivalent to refuted sentences.

\subsection{The Weak Logical Rules}

Let's comment on and justify the weak logical rules, i.e. the top rule and the bottom rule. They are logical since they realize through an information transformation process the presumed logical content of the logical connectives top and bottom. Note that, in the foundational perspective of $E C L$, without such rules, the presence in the system of the mentioned o-typed logical connectives would be not motivated and they should be excluded from the language. On the other side, they are the only rules through which not explicitly constructed logical information can be introduced in the argumentative discourse. Indeed, they represent a very constrained and regulated way to partially have that information introduction power of the standard weakening rule. This is also why they are called weak, and their principal formulas are qualified as weakly introduced. This causes inferential limitations. If $\perp \vdash C$ and $D \vdash \top$ are the conclusions of any bottom rule and top rule respectively, we cannot apply to them a $\supset-L$ rule and infer $C \supset D, \perp \vdash \top$, since both the auxiliary formulas are weakly introduced. As a matter of fact $\perp \vdash \perp$ and $T \vdash T$ are not logical axioms, they are conclusions of weak logical rules, so that one of the two cedents ([1] p. 10) is always weakly introduced. Nevertheless, the contribution of weak logical rules to $E C L$ inference is substantial; otherwise the information sources of ECL proofs would be too poor. In addition, their weakly introduced principal formula can anyway contribute to infer strongly introduced formulas: from $D \vdash T$, we can infer $\vdash$ $D \supset \top$ that is a strongly introduced formula, as the principal formula of a $\supset-R$ must be. We shall prove in Section 6 that weak logical rules must only occur as the initial rule in a branch: in addition, their principal formula has not auxiliary formula, so that it has no predecessor. This justifies the requirement that formulas $\forall x_{o}\left(x_{o}\right), \exists y_{o}\left(y_{o}\right)$ do not occur as sub-formulas of the principal formula of any weak logical rule. In a $\mathbf{Z}_{\omega}$-proof, $\forall x_{o}\left(x_{o}\right), \exists y_{o}\left(y_{o}\right)$ mark elimination judgments about previously produced logical information. Then, their occurrence is meaningless in an initial (uppermost) formula of the proof tree.

\subsection{The Exclusion of Equational Logic $\mathrm{EQ}_{\omega}$ from $\mathrm{Z}_{\omega}$}

In $\mathbf{L K}_{\boldsymbol{\omega}}$ and $\mathbf{L} \mathbf{J}_{\boldsymbol{\omega}}$, Equational Higher Order Logic $\mathbf{E} \mathbf{Q}_{\boldsymbol{\omega}}$ can be fully included in the system and works together with the logical part. For the $\mathbf{E Q}_{\boldsymbol{\omega}}$-axioms see [7], Sec. 2.4. What must be clearly emphasized is that in the higher order context and inside the Church formalism $\mathbf{E} \mathbf{Q}_{\boldsymbol{\omega}}$ becomes extremely powerful and mixes itself with the logical connectives' deduction action. This is clear if we consider that each theorem $B$ of $\mathbf{L} \mathbf{K}_{\boldsymbol{\omega}}$ and $\mathbf{L} \mathbf{J}_{\boldsymbol{\omega}}$ can be provably constricted to the atomic formula $B={ }_{o} \top$, and that the equality predicate on type $o$ can 
be provably identified with the logical equivalence between propositions, i.e., in the $E C L$ perspective, between judgments. From the standpoint of $E C L$, aiming to obtain a very fine characterization of logical connectives through a constructive approach, this is not admissible. Equational Logic is explicitly excluded from $E C L$. More generally, we point out that, in the context of explicit constructivism, is also inadmissible the confusion between the equality relation $=_{o}$ and the logical implication $\supset$ or double-implication $\longleftrightarrow$. We think that the equality relation can be only defined a priori in a platonic universe. In a knowledge representation setting, we are not able to imagine an epistemic subject that, inside an empirical world and through an effective process can establish that two objects are equal, with the same meaning owned by the statement "these two Euclidean triangles are equal" affirmed inside a platonic universe. On the contrary, if we consider the epistemic subject that formulates judgments on the world, the implication or double-implication relation must be established by a construction which increases the complexity of the judgment through the logical rules, starting from elementary data. Observe that in $\mathbf{Z}_{\omega}$ theorems that are atomic formulas do not exist (with the minor exception of possible $\beta$-redexes, which are a bit artificial form both for possible judgments and for possible data), coherently with the principle that elementary data cannot be judgments. Differently, Equational Logic $\mathbf{E Q}_{\boldsymbol{\omega}}$ produces a multitude of atomic theorems, most of them having a substantial and non-artificial information content, such as, for example, the assertion $B={ }_{o} F \wedge \neg F$, establishing that the arbitrarily complex formula $B$ is logically equivalent to a contradiction.

\subsection{The Strong Logical Rules}

The originality of the proposed logic is mainly expressed in these rules. In fact, the constraints involving these rules are not local, i.e. they do not operate on the occurrence of the specific rule $\mathcal{R}$ in a proof, but are conditions concerning the whole proof $P$ in which the rule occurs: to apply $\mathcal{R}$ it is necessary, in general, to examine all the introductions of the uppermost ancestors of the auxiliary formulas of $\mathcal{R}$ in the proof segment of $\mathrm{P}$ standing above the $\mathcal{R}$-premise(s). We deem that relevant innovations in Logic could be obtained by changing the praxis of imposing only local constraints on a single rule. This lightly changes the usual notion of performing a proof, and could produce innovative results and situations, perhaps more than the introduction of new connectives and new rules. By recalling the Definitions of Section 3, the distinction between strongly introduced formula in a proof $\mathrm{P}$ and weakly introduced formula in a proof $\mathrm{P}$ should result natural. Axioms are the choices of the epistemic subject, on which it decides to found its reasoning. Weakening and weak logical rules are auxiliary tools, useful to introduce information. On the other hand, a proof without at least one axiom occurrence cannot exist, while infinite proofs may exist without weakening or weak rule occurrences. It must be emphasized that the minor strong logical rules $\vee-R, \wedge-L$ are differently presented w.r.t. the usual standard presentation, that is both the auxiliary formulas must occur as isolated in the premise. This reflects two crucial requirements. First, if this would not be the case, the introduction of one of the maximal disjunct (conjunct) of the principal formula 
would be an arbitrary hidden weakening. Furthermore, since we need to constraint all the uppermost ancestors of both the auxiliary formulas in the whole proof-segment above, both the formulas must explicitly occur in the premise.

\subsection{The Structures of Weakening and Cut in $\mathbf{Z}_{\omega}$}

The constraints on the weakening rule, i.e. the imposition on principal formulas to be atomic, should be quite clear. It is at the basis of Explicit Constructive Logic: only elementary data can be used without having been constructed. A first non trivial fact follows immediately, thus clarifying the differences from $\mathbf{L} \mathbf{K}_{\boldsymbol{\omega}}$ and $\mathbf{L} \mathbf{J}_{\boldsymbol{\omega}}$ : if $X \vdash Y$ is $\mathbf{Z}_{\omega}$-provable, its over-sequents $U \vdash V$ with $X \subset U$ and $Y \subset V$, are, in general, not $\mathbf{Z}_{\omega}$-provable. A motivation of the presence of two rules $W 1$ and $W 2$ is due: while $\mathrm{W} 1$ is immediately understandable, less obvious is the necessity of $W 2$, i.e. of arbitrary weakenings on the empty sequent. The reason arises from the fact that $\mathbf{Z}_{\omega}$ is supposed to be possibly extended to various (countably many) axiomatized theories $\mathbf{T}_{\mathbf{j}}$ 's. Some of them are expected to be absolutely inconsistent, and we usually identify this situation with the provability of the empty sequent. But this does not work in the $E C L$-framework, since from the empty sequent, $\mathbf{Z}_{\omega}$-rules minus $W 2$, in general, cannot derive all formulas of the language as theorems. Therefore, $W 2$ is added. The motivations of $C u t 2$ are also linked to the ones of $W 2$ : if a $\mathbf{Z}_{\omega}$-based theory $\mathbf{T}$ has any non atomic theorem and any non atomic refuted that are identical, i.e. $\mathbf{T}$ proves both $B \vdash$ and $\vdash B, \mathbf{Z}_{\omega}$-rules minus $C u t 2$, in general, cannot derive the empty sequent. Thus, $C u t 2$ is added. We consider now the substantial restrictions that are imposed to the cut rule. They reflect the requirements already considered about the introduction and the elimination of logical information by a reasoning epistemic subject: logical information is not eliminated by a material deletion, but only by a logical transformation. The selection of logical information is a thinking act, requires elaboration, then it must involve logical rules. Thus, cut at most deletes atomic formulas, i.e. elementary data. On the other hand, the peculiar structure of the cut rule cannot become a clandestine arbitrary weakening, and this is obtained by imposing the same context for the two premises.

\section{Possible New Effective Applicability of ECL-based Higher Order Logic}

It is well known that, in full Higher Order Logic, the unbounded quantification on $o$-typed formulas, or on formulas of arbitrary types where $o$-typed formulas occur as Church-subterms, makes it extremely difficult to establish any useful link between the formulas occurring in the root of a cut-free proof-tree $\mathrm{P}$ and the formulas occurring in the overstanding sequents alongside the branches of $\mathrm{P}$.

To find how to exploit the information and proof power of higher order quantification, without chaotic or collapsing phenomena, is a main goal of our constructive view of higher order logic. The envisaged road is the following:

to suitably and lightly normalize higher order quantification by a set of constraints allowing a Normal Form Theorem for the proofs of an adequately expressive sub-system of $\mathbf{L K}_{\boldsymbol{\omega}}$. 
A Normal Form Theorem (NFT) for a system V (see [1] or for example [5] where a NFT for Arithmetic is proposed) is characterized by:

i) an effective description of the transformation of a $\mathbf{V}$-proof into a $\mathbf{V}$-proof tree with the same root, partitioned in blocks such that each block includes only homogeneous rules or axioms;

ii) a set of effective procedures such that given a root sequent $L$, the following reasonable estimates about the features of a possible proof $\mathrm{Q}$ of $L$ in $\mathbf{V}$ can be produced:

an estimate of the possible $\mathbf{V}$-rule instance set occurring in $\mathrm{Q}$

an estimate of the possible $\mathbf{V}$-axiom instance set occurring in $\mathrm{Q}$

an estimate of the length and the width of $\mathrm{Q}$

an estimate of the formula (or term) set occurring in Q.

It is quite clear that an efficient Normal Form Theorem for an adequate and non redundant $\mathbf{L} \mathbf{K}_{\boldsymbol{\omega}}$ sub-system can give a new basis for higher order automated deduction and knowledge representation. We believe that:

the very strong and peculiar ECL constructivity of the system $\mathbf{Z}_{\omega}$ allows us to state those normative constraints on its quantification power that can generate an adequately expressive subsystem $\mathbf{Z}_{\omega}^{*}$ that admits a Normal Form Theorem. We present now a first hint of the system $\mathbf{Z}_{\omega}^{*}$ that should exemplify how further constructive conditions imposed on the quantification judgements of the epistemic subject may lead to a Normal Form Theorem. As a basic feature of $\mathbf{Z}_{\omega}^{*}$ we introduce the notion of $\mathbf{Z}_{\omega}$-proof with the witness property. We previously recall that the language includes, for each type $\gamma, \aleph_{0}$ free variables (atoms) $\left\{b_{\gamma}^{j}\right\}$, univocally individuated by their index $j \in \mathbf{N}$, and $\aleph_{0}$ bound variables (atoms) $\left\{y_{\gamma}^{i}\right\}$, univocally individuated by their index $i \in \mathbf{N}$ (see [7] Section 2.2). In the following definition, the most relevant point is b):

Definition 4. A $\mathbf{Z}_{\omega}$-proof $\mathrm{P}$ has the witness property if the following conditions hold:

a) Each time $o$-typed formulas/terms $B_{o}^{j}, j=1, \ldots, m, m \geq 1$ occur in $\mathrm{P}$ as the auxiliary formula $E$ of a Comp rule, or are included in it as sub-terms, then the bound variable $z_{o}$ in the corresponding principal formula $\mathcal{Q} z_{o}\left(z_{o}\right), \mathcal{Q} \in\{\forall, \exists\}$ has as index the gödel number ([7] Section 2.5) of their sequence.

b) Any auxiliary formula of a Comp rule in $\mathrm{P}$ may have $o$-typed sub-terms only if the quantification is over the type $o$.

c) In $\mathrm{P}$ isolated formulas of the form $a_{o}, b_{o}$, i.e. free variables of type $o$ which occur as isolated formulas in a sequent, cannot be auxiliary formulas of quantifier rules.

Proposition 1. The property "to be a proof with the witness property of the system $\mathbf{Z}_{\omega}$ " is a recursive relation, it can be expressed by a recursive predicate inside Primitive Recursive Arithmetic PRA and is a decidable property.

Proof. The Definition above describes exactly effective conditions to get a $\mathbf{Z}_{\omega}-$ proof with the witness property.

Only as one of the possible example of the effective control on proofs allowed by the witness property we mention these results: 
Proposition 2. a) Let $P$ be a proof in $\mathbf{Z}_{\omega}$ with the witness property. If in $P$ a formula of the form $\mathcal{Q} z_{o}\left(z_{o}\right), \mathcal{Q} \in\{\forall, \exists\}$ is introduced, then in the root at least one formula of the form $\mathcal{H} z_{o}\left(z_{o}\right), \mathcal{H} \in\{\forall, \exists\}$ occurs.

b) Let $P$ be a proof in $\mathbf{Z}_{\omega}$ with the witness property. Let us suppose that quantified formulas over the type o do not occur in the P-root. Then each atom of type o occurring in $P$ occurs also in the root of $P$.

Definition 5. The weakly normalized system $\mathbf{Z}_{\omega}^{*}$ is so defined: a) Axioms, propositional rules, structural rules of $\mathbf{Z}_{\omega}^{*}$ are the same as that of $\mathbf{Z}_{\omega}$ and with the same constraints; b) Quantifier rules of $\mathbf{Z}_{\omega}^{*}$ have the same constraints as the ones of $\mathbf{Z}_{\omega}$, and moreover are applied in a proof-tree in a way such that the resulting proof has the witness property, i.e. it fulfils all the conditions stated in the previous Definition of the witness property. c) The $\lambda$-rule is omitted from the deduction aparatus.

Therefore, in $\mathbf{Z}_{\omega}^{*}$ all proofs have the witness property. Thus, even if the higher order quantification power is not dramatically bounded at all, some interesting links between the formulas occurring in the root and the rule instances and formulas occurring in the above proof-segments are at disposal. We will see such links at work in particular in Section 7.

\section{Elementary Proof-theory and Expressivity of $\mathrm{Z}_{\omega}$}

Proposition 3. $\mathbf{Z}_{\omega}$ admits cut-elimination.

Proof. The proof is straightforward. Indeed, Cut1-formulas can be only atomic. Then they can be introduced in any $\mathbf{Z}_{\omega}$ - proof only by weakenings or by logical axioms. This makes the proof-reductions to get cut-elimination very easy. As to $C u t 2$ the set of $C u t 2$-occurrences in the $\mathbf{Z}_{\omega}$-proofs is empty, due to the consistency of $\mathbf{Z}_{\omega}$.

In the sequel we assume to work only with cut-free $\mathbf{Z}_{\boldsymbol{\omega}}$-proofs. Moreover, by coherence with $E C L$ setting, we will consider only the equality free versions of type theories $\mathbf{L} \mathbf{K}_{\boldsymbol{\omega}} \mathbf{L} \mathbf{J}_{\boldsymbol{\omega}}$, that so have the full cut elimination property. The next results could seem to have obvious proofs. This would be a misunderstanding, since in $E C L$ the form of the logical rules is essentially standard, but their application conditions are not standard at all. For example, if $F$ is not atomic, then $F \vdash F$ is not a $\mathbf{Z}_{\boldsymbol{\omega}}$-logical axioms, and, in general, nobody can easily assert or deny that it must be a $\mathbf{Z}_{\boldsymbol{\omega}}$-theorem.

Proposition 4. $\mathbf{Z}_{\omega}$ proves $\aleph_{0}$ instances both of the excluded middle principle $B \vee \neg B$ and of the left double negation principle $\neg \neg B \supset B$ that Intuitionistic Higher Order Logic $\mathbf{L} \mathbf{J}_{\boldsymbol{\omega}}$ does not prove.

Proof. Let $G^{+}, H^{+}$different non logical constants of type $o$. It is easy to see that $\mathbf{Z}_{\omega}$ proves the sequent $G^{+} \wedge H^{+} \vdash G^{+} \wedge H^{+}$with all the formulas strongly introduced in the proof. Then we produce in $\mathbf{Z}_{\omega}$ the following proof segment: 


$$
\frac{\frac{G^{+} \wedge H^{+} \vdash G^{+} \wedge H^{+}}{\vdash G^{+} \wedge H^{+}, \neg\left(G^{+} \wedge H^{+}\right)} \neg-R}{\vdash\left(G^{+} \wedge H^{+}\right) \vee \neg\left(G^{+} \wedge H^{+}\right)} \vee-R
$$

where all the rules have strongly introduced auxiliary formulas. Differently, by applying the cut-elimination property of $\mathbf{L} \mathbf{J}_{\boldsymbol{\omega}}$ it is evident that $\mathbf{L} \mathbf{J}_{\boldsymbol{\omega}}$ cannot prove the same end-sequent without breaking the local constraints of each $\mathbf{L} \mathbf{J}_{\boldsymbol{\omega}}-$ rule, imposing at most one formula in each succedent. Analogous considerations hold for the sequent $\vdash \neg \neg H^{+} \supset H^{+}$. Moreover, the thesis can be easily extends to arbitrarily complex instances of the mentioned principles.

Proposition 5. $\mathbf{Z}_{\omega}$ proves $\aleph_{0}$ instances of the non contradiction principle $\neg(A \wedge$ $\neg A)$ that equality free paraconsistent type theory $\mathbf{C I}_{\omega}$, extending the system $\mathbf{C I}$, cannot prove.

Proof. Let $B^{+}$be an atomic formula. It is wellknown that $\mathbf{C I}_{\omega}$ does not prove $\vdash \neg\left(B^{+} \wedge \neg B^{+}\right)$(for the details on CI see [6]). The following proof can be produced in $\mathbf{Z}_{\omega}$ :

$$
\frac{\frac{\frac{B^{+} \vdash B^{+}}{B^{+}, \neg B^{+} \vdash} \neg-L}{B^{+} \wedge \neg B^{+} \vdash} \wedge-R}{\vdash \neg\left(B^{+} \wedge \neg B^{+}\right)} \neg-R
$$

where all the auxiliary formulas of the rules are strongly introduced.

In the following, we simply state some lemmas without proofs:

Lemma 1. The weak logical rules top rule and bottom rule can be only initial rules in a proof branch, i.e. they always occur as the uppermost rules of the branch.

Lemma 2. Let $Q$ be a proof in $\mathbf{Z}_{\omega}$ with root $X \vdash Y, B$ where $B$ is the integral descendant of the principal formulas of a set $W \equiv\left\{\frac{\perp \vdash}{\perp-B} \mathcal{R}_{j}\right\}$ of bottom rules in $Q$. Then we can replace each element of $W$ in $Q$ with the axiom $\perp \vdash$ obtaining a proof $P$ of $X \vdash Y$ in $\mathbf{Z}_{\omega}$.

Lemma 3. Analogous to the last Lemma, by replacing "bottom rule" with "top rule" and the formula bottom $\perp$ with the formula top $\top$.

\section{What ECL does not want to prove: $\mathrm{Z}_{\omega}^{*}, \mathrm{Z} 1, \mathrm{ZP}$ as paraconsistent and pseudo-intuitionistic systems}

A very remarkable property of $E C L$ is that without imposing any local constraint on negation rules of its systems, it nevertheless shows simultaneously a relevant and interesting intuitionistic and paraconsistent behaviour of its proofs. We will use now the weakly normalized system $\mathbf{Z}_{\omega}^{*}$ (Definition 5) that is a convenient setting of our epistemic consideration. We have the following results ${ }^{3}$ :

\footnotetext{
$\overline{3}$ in the sequel the superscript $(.)^{+}$in atomic formulas will be omitted
} 
Theorem 6. Consider the following instance of non contradiction principle expressed by the sequent $S: \vdash \neg[(\perp \wedge(B \wedge C)) \wedge \neg(\perp \wedge(B \wedge C))]$ where $B$ and $C$ are different o-typed non logical constants. Then $S$ is not $\mathbf{Z}_{\omega}^{*}$-provable. Since $S$ belongs also to propositional and first order languages the same holds for $\mathbf{Z} \mathbf{1}$ and $\mathbf{Z P}$.

Proof. Suppose ad absurdum that $S$ is the root of a proof $\mathrm{Q}$ of $\mathbf{Z}_{\omega}^{*}$. If the root formula $B$ is also the integral descendant of the principal formula of a set of of bottom rule occcurences in Q, by Lemma 2 we delete such rules and get a proof Q' where the root formula $B$ is never introduced by a bottom rule occurrence: indeed, we exclude that the root of Q' could result the empty sequent, by the absolute consistency of $\mathbf{Z}_{\omega}^{*}$. Therefore, by properties of $\mathbf{Z}_{\omega}^{*}$, the end rule of $\mathrm{Q}^{\prime}$ must be a $\neg-L$ rule having the sequent $M \equiv(\perp \wedge(B \wedge C)) \wedge \neg(\perp \wedge(B \wedge C)) \vdash$ as premise, and let $H$ be its root formula. In $M$ top formulas do not occur: then, by Proposition 2 (item $b$ )), in Q' neither top formulas nor top rules can occur. Thus, neither $H$ nor $H$ sub-formulas can be integral descendant of principal formulas of top rule occurrences in Q'. $H$ must be so the conclusion of a $\wedge-L$ rule, with premise $K \equiv(\perp \wedge(B \wedge C)), \neg(\perp \wedge(B \wedge C)) \vdash$. By analogous reasons $K$ is the conclusion of a $\neg-R$ rule with premise $N \equiv(\perp \wedge(B \wedge C)) \vdash$ $(\perp \wedge(B \wedge C))$. Let $D$ be the succedent of $N$. We have to examine the possibility that $D$ has been introduced by bottom rules in Q'. We have two possible cases. The first one is that $D$ is exclusively the integral descendant of principal formulas of bottom rule occurrences in Q'. By Lemma 2 we delete them, and get $G \equiv$ $\perp \wedge(B \wedge C) \vdash$ as the root of a $\mathbf{Z}_{\omega}^{*}$-proof W. Since top rules do not exist in W, the premise of $G$ in W must be $\perp, B \wedge C \vdash$, that necessarily has $\perp, B, C \vdash$ as premise: this is absurd, since being both $B$ and $C$ obviously weakly introduced, they cannot be auxiliary formulas of a $\wedge-L$ rule. The second case is that $D$ is not only the integral descendant of principal formulas of bottom rule occurrences so that, having deleted these by Lemma 2, we obtain a proof $\mathrm{V}$ of the sequent $L \equiv \perp \wedge(B \wedge C) \vdash \perp \wedge(B \wedge C)$ where no cedent is the integral descendant of principal formulas of weak logical rule occurrences. $L$ must be the conclusion of a strong logical rule. Indeed, suppose that the end rule of $\mathrm{V}$ is any $\wedge-R$ rule. Then its premises, that are both necessarily $\mathbf{Z}_{\omega}^{*}$-provable, are $J 1 \equiv \perp \wedge(B \wedge C) \vdash$ $\perp$ and $J 2 \equiv \perp \wedge(B \wedge C) \vdash(B \wedge C)$ : unfortunately, it is evident that the succedent of $J 1$ cannot be strongly introduced, so that the $\wedge-R$ constraint would not be respected. We must so assume that the end rule of $\mathrm{V}$ is any $\wedge-L$ rule, with premise $J 3 \equiv \perp, B \wedge C \vdash \perp \wedge(B \wedge C)$. J3 can be the conclusion either of a $\wedge-L$ or of a $\wedge-R$. In the first case the possible premise is $J 4 \equiv \perp, B, C \vdash \perp \wedge(B \wedge C)$, in the second case the two possible premises, both necessarily $\mathbf{Z}_{\omega}^{*}$-provable, are $J 5 \equiv \perp, B \wedge C \vdash \perp$ and $J 6 \equiv \perp, B \wedge C \vdash B \wedge C$. However, the sucedent of $J 5$ can be only weakly introduced and the same problems observed for $J 1$ stop the examined possibility. Thus, we have to examine the possible provability of $J 4$. The possibility that $J 4$ is the conclusion of any $\wedge-R$ rule gives the same problems already noted for $J 1$ and $J 5$. We have then to suppose that the succedent of $J 4$ has been introduced in $\mathrm{V}$ only by a set of bottom rule occurrences, and that the $\perp$ in the $J 4$-antecedent is the integral descendant of the premise formulas 
of such bottom rules. By Lemma 2, we delete such bottom rule occurrences and get a proof $\mathrm{Z}$ of the sequent $J 7 \equiv \perp, B, C \vdash$ where by construction $B, C$ are the only integral ancestors of the auxiliary formulas of the $\wedge-L$ with conclusion $J 3 \equiv \perp, B \wedge C \vdash \perp \wedge(B \wedge C)$. But this is absurd, since both $B$ and $C$ in $J 7$ would be only introduced by weakenings in $\mathrm{V}$, i.e. both are weakly introduced, and the $\wedge-L$ constraints would not be respected ${ }^{4}$.

Theorem 7. Consider the following instance of excluded middle principle expressed by the sequent $M: \vdash\left[\top \vee \exists x_{i} B\right] \vee \neg\left[\top \vee \exists x_{i} B\right]$ with $B$ o-typed non logical constant. Then $M$ is not $\mathbf{Z}_{\omega}^{*}$-provable. Since $M$ belongs also to the first order language the same holds for $\mathbf{Z 1}$.

\section{Conclusions}

The introduction of the ECL logic and the first results regarding its relations with intuitionistic and paraconsistent logics are the main topics of the paper. We stressed the higher order setting since we believe that it is a relevant issue to find ways to make HOL a foundational setting for knowledge representation (constructive and with controlled use of instantiations). The future work will be devoted to further analyze the characteristics of ECL. As some considerations already present in the paper suggest the constructivity of ECL is not related to the "not rules" but to some peculiarities of the implication and of the possible proofs that can be accepted. These properties should be relevant for the use of ECL as the foundation logic of both Logic Programming and Theorem Proving.

\section{References}

1. S.R. Buss (Ed.), Handbook of Proof Theory, Elsevier, Amsterdam, 1998.

2. W. A. Carnielli, M. E. Coniglio, J. Marcos, 'Logics of formal inconsistency' in Handbook of Philosophical Logic, D. Gabbay, F. Guenthner (Eds.), 2nd ed., volume 14, Kluwer, Dordrecht, 2005.

3. A. Church, 'A Formulation of Simple Theory of Types', Journal of Symbolic Logic, $5,1940,56-68$.

4. M. De Marco, J. Lipton, 'Completeness and Cut-elimination in the Intuitionistic Theory of Types', Journal of Logic and Computation, 15, 2005, 821-854.

5. P. Forcheri, P. Gentilini, M.T. Molfino, 'Informational Logic in knowledge representation and automated deduction', AI COMMUNICATIONS, vol. 12,1999, 185208.

6. P. Gentilini, 'Proof Theory and Mathematical Meaning of Paraconsistent CSystems', Journal of Applied Logic, vol. 9, 3, 2011, 171-202.

7. P. Gentilini, M. Martelli, 'Abstract Deduction and Inferential Models for Type Theory', Information and Computation, vol. 208, Issue 7, July 2010, 737-77.

8. D. Miller, G. Nadathur, F. Pfenning, and A. Scedrov, 'Uniform Proofs as a Foundation for Logic Programming'. Annals of Pure and Applied Logic 51, 1991, 125-157.

9. G. Takeuti, Proof Theory, North-Holland, Amsterdam, 1987.

10. A.S. Troelstra, D. van Dalen, Constructivism in Mathematics, Vol.1, Elsevier, 1988.

${ }^{4}$ It is not difficult to imagine that infinitely many sequents having a form similar to $S$ are not $\mathbf{Z}_{\omega}^{*}$-provable. 